Verification Test Suite (VERTS) For Rail Gun Applications using ALE3D: 2-D Hydrodynamics \& Thermal Cases

F. M. Najjar, J. Solberg, D. White

April 25, 2008 
This document was prepared as an account of work sponsored by an agency of the United States government. Neither the United States government nor Lawrence Livermore National Security, LLC, nor any of their employees makes any warranty, expressed or implied, or assumes any legal liability or responsibility for the accuracy, completeness, or usefulness of any information, apparatus, product, or process disclosed, or represents that its use would not infringe privately owned rights. Reference herein to any specific commercial product, process, or service by trade name, trademark, manufacturer, or otherwise does not necessarily constitute or imply its endorsement, recommendation, or favoring by the United States government or Lawrence Livermore National Security, LLC. The views and opinions of authors expressed herein do not necessarily state or reflect those of the United States government or Lawrence Livermore National Security, LLC, and shall not be used for advertising or product endorsement purposes.

This work performed under the auspices of the U.S. Department of Energy by Lawrence Livermore National Laboratory under Contract DE-AC52-07NA27344. 


\title{
Verification Test Suite (VERTS) For Rail Gun Applications using ALE3D: 2-D Hydrodynamics \& Thermal Cases *
}

\author{
Fady Najjar $†$ Jerome Solberg $\ddagger$ Daniel White ${ }^{\S}$
}

April 25, 2008

\begin{abstract}
A verification test suite has been assessed with primary focus on low reynolds number flow of liquid metals. Thid is representative of the interface between the armature and rail in gun applications. The computational multiphysics framework, $A L E 3 D$, is used. The main objective of the current study is to provide guidance and gain confidence in the results obtained with $A L E 3 D$. A verification test suite based on $2-\mathrm{D}$ cases is proposed and includes the lid-driven cavity and the Couette flow are investigated. The hydro and thermal fields are assumed to be steady and laminar in nature. Results are compared with analytical solutions and previously published data. Mesh resolution studies are performed along with various models for the equation of state.
\end{abstract}

\section{Introduction}

Our primary focus in the present study is to verify the multiphysics hydrodynamics code, ALE3D, for fluid and thermal flow problems relevant to rail-gun based applications. First, we define the following two terms:

- Verification: "the process of demonstrating on the basis of evidence that the underlying mathematical equations are solved correctly for the intended application"

- Validation: "the process of demonstrating on the basis of evidence that the underlying mathematical equations are correct for the intended application" [7].

More formal definitions are discussed in Oberkampf \& Trucano [5] and Oberkampf, Trucano \& Hirsch [6].

The rail gun application is a complex multiphysics system with mass transformation, phase change, energy release, deformations, to name a few. The canonical railgun consists of a pair of straight metal rails held fixed by a an insulating containment superstructure, and a metal armature that slides between the two rails. The contact force is large, the velocities are high, and there is evidence that a thin layer of melted aluminum quickly forms in the interface. Hence understanding this metal-liquid-matel interface is important to railgun designers. For this particular Verfication Test Suite (VERTS), several test cases have been identified where hydrodynamics and thermal phenomena occur similar to those observed in rail gun systems. These cases include the 2-D steady laminar lid-driven cavity and the 2-D steady laminar

${ }^{*}$ This work was performed under the auspices of the U.S. Department of Energy by Lawrence Livermore National Security under contract No. DE-AC52-07NA27344

${ }^{\dagger}$ B-Division, Lawrence Livermore National Laboratory (LLNL), najjar2@1lnl.gov

$\ddagger$ Defense Technologies Engineering Division, LLNL, solberg2@llnl.gov

$\S$ Engineering Technology Division, LLNL, white37@llnl.gov 
Couette flow with thermal effects. Currently, the flow complexity is kept to only include laminar flow (low Reynolds number) and steady-state behavior. Effects of turbulence and flow unsteadiness will be more relevant in a validation study, i.e. experimental data is required. In this VERTS we intentionally neglect electromagnetic effects, this will be the subject of follow-on VERTS.

The present report is organized as follows. Section 2 presents the governing equations along with the mathematical formulation. Section 3 describes the results for the 2-D lid-driven cavity in steady-state mode for a variety of liquids including air and melted metals. The results for the 2-D Couette flow with and without thermal transport are discussed in Section 4. Conclusions and summary are outlined in Section 5 .

\section{Governing Equations \& Mathematical Formulation}

ALE3D is a three-dimensional (3-D) magneto-thermal-hydrodynamics (MHD) simulation software using an arbitrary Lagrangian-Eulerian (ALE) approach. It has been developed at LLNL over the past 30 years and is currently used for a variety of applications including thermal explosions, weapon penetration, thermal cook-offs and void collapse, to name a few. The governing equations consist of conservation of mass, momentum and energy, written as follows:

$$
\begin{array}{r}
\frac{d \rho}{d t}=-\rho \vec{\nabla} \cdot \vec{u} \\
\rho \frac{d \vec{u}}{d t}=\vec{\nabla} \cdot \mathbf{T}+\vec{j} \times \vec{B} \\
\frac{d \epsilon}{d t}=\frac{1}{\rho} \operatorname{Tr}\left(\mathbf{E}_{T o t} \cdot \mathbf{T}\right)+\frac{1}{\rho} \vec{j} \cdot \vec{E}
\end{array}
$$

where $\rho, \vec{u}$ and $\epsilon$ represent the density, velocity vector and internal energy, respectively. T corresponds to the total stress tensor and is separated into its two irreducible components:

$$
T_{i j}=S_{i j}+p \delta_{i j}
$$

where $p$ is the isotropic pressure defined as $p=-\frac{1}{3} \operatorname{Tr}(\mathbf{T})$ and $S_{i j}$ are the stress deviators. $E_{T o t, i j}=$ $\frac{1}{2}\left(\frac{\partial u_{i}}{\partial x_{j}}+\frac{\partial u_{j}}{\partial x_{i}}\right)$ represents the total strain rate tensor and the Lagrange time derivative, $\frac{d}{d t}$, corresponds to $\frac{\partial}{\partial t}+\vec{u} \cdot \vec{\nabla}$.

In MHD applications, $\vec{B}$ and $\vec{E}$ represent the magnetic and electric vector fields. Further, for the current cases considered, the Navier-Stokes equations are invoked; hence the viscous stresses and viscous dissipation terms are included in the conservation of momentum and energy.

The governing equations are discretized using a hybrid finite-element/ finite-volume method (FEM/FVM) approach and are solved in a weak form. The multiphysics software, ALE3D, is used to performed these simulations. Typically, the node coordinates and velocities are node-centered variables; while the density, pressure, internal energy, temperature, etc are zone-centered quantities. A zone is a cell or element on a discretized mesh. Proper basis functions are defined based on tri-linear (bi-linear for 2-D) and piecewise constant. The time-integration scheme is based on a leap-frog method. Further details are available in $A L E 3 D$ User's manual [1]. A variety of equations of state (EOS) are currently available in $A L E 3 D$ including the $\gamma$-law for ideal gases, Grüneisen strength models, and tabulated EOS (e.g. LEOS, SESAME). 


\section{Verification Test Case for Hydro Applications: 2-D Steady Laminar Flow for Lid-Driven Cavity}

The first test case considered for VERTS pertaining to rail gun applications is the 2-D steady lid-driven cavity. The geometry consists of a square cavity where its top wall is being moved at a constant velocity while the remaining walls are held fixed. The problem considered is a canonical case to verify computational codes. A vast body of literature spanning over 20 years is available for detailed comparison. These studies include the work of Ghia et al. [4], Deng et al. [3], and Botella \& Peyret [2], to name a few.

In this section, working liquids with disparate strength models are considered and include air and melted metals such as aluminum and copper. A mesh resolution analysis is also performed. The study is performed for several Reynolds numbers, 100 and 1000. The Reynolds number is defined as $R e=\frac{\rho U_{t o p} L}{\mu}$, where $\rho$ refers to the fluid density, $U_{\text {top }}$ represents the velocity of the top wall, and L corresponds to the cavity length and height; while $\mu$ is defined as the kinematic viscosity. At the Reynolds number considered, the flow remains 2-D, laminar and steady. The governing equations in $A L E 3 D$ are integrated in time to reach a quasi-steady state. We will present our approach on how to achieve a quasi-steady state in the context of an unsteady solver.

Table 1 summarizes 8 test cases with the Reynolds numbers and corresponding mesh resolutions to verify the 2 -D steady laminar flow for the lid-driven cavity.

\begin{tabular}{cc}
\hline Re & Mesh Resolution \\
\hline 100 & $32 \times 32,64 \times 64$, \\
& $128 \times 128,256 \times 256$ \\
1000 & $64 \times 64,128 \times 128$, \\
& $256 \times 256,512 \times 512$ \\
\hline
\end{tabular}

Table 1: Simulation matrix considered to verify for the 2-D lid-driven cavity.

\subsection{Air with $\gamma$-Law: $\operatorname{Re}=100$}

Most published studies are based on an incompressible fluid assumption (where the mach number is much less than $1, M a<<1$ ) at various Reynolds numbers. Since the MHD capability is currently active with the explicit compressible hydro component of ALE3D, verification of the compressible hydro component is performed. As an initial step to mimick incompressible liquids with no strength, air is considered an ideal gas with $\gamma=1.4$.

The Reynolds number is defined as $R e=\frac{\rho_{\text {Air }} U_{\text {top }} L}{\mu}$, where $\rho_{\text {Air }}$ refers to air density, $U_{\text {top }}$ represents the velocity of the top wall, and L corresponds to the cavity length and height; while $\mu$ is defined as the kinematic viscosity. Further, to properly compare with published studies performed on incompressible flows, the compressibility effect has to kept low. In all the cases studied, a Mach number was at a value of $\sim 5.0 \times 10^{-3}$. The problem configuration consists of a square cavity with length of $\mathrm{L}=1 \mathrm{~cm}$. The flow properties are $\rho=10^{-3} \mathrm{~g} / \mathrm{cm}^{3}$ and $\mu=10^{-8} \mathrm{~g} / \mathrm{cm} / \mu s$. For a specified Reynolds number (Re), a top-wall 
velocity, $\mathrm{U}_{\text {top }}$, is calculated. The spatial and velocity scales are based on $\mathrm{L}$ and $\mathrm{U}_{\text {top }}$. Simulations are performed for several mesh resolutions consisting of $32 \times 32,64 \times 64,128 \times 128$ and $256 \times 256$ cells. In this section, computations with $\mathrm{Re}=100$ are considered. All results presented have been nondimensionalized appropriately by the cavity length and the top wall velocity.

$A L E 3 D$ integrates the governing equations using an explicit time integration approach. To insure that the flow field reached a quasi-steady state, the time evolution of a representative global quantity is monitored. The normalized kinetic energy, defined as $k e_{n o r m}=\left(u^{2}+v^{2}\right) / U_{\text {top }}^{2}$, is selected and its time variation is shown in Fig. 1. It is clearly seen that the flow has reached a quasi-steady state where the values reach a plateau and change within $10^{-6}$ for the various mesh sizes after $t=10000 \mu \mathrm{s}$. Once the flow achieves a quasi-steady state, several flow features are extracted. The distributions of the $u$ $(v)$-velocity along the vertical (horizontal) centerline are shown in Fig. 2(a) and (b) for the 4 meshes considered. Also plotted are the results obtained by Ghia et al. [4]. It is seen that the agreement with the published results is qualitatively quite good. We will present below more quantitative analysis at select locations..

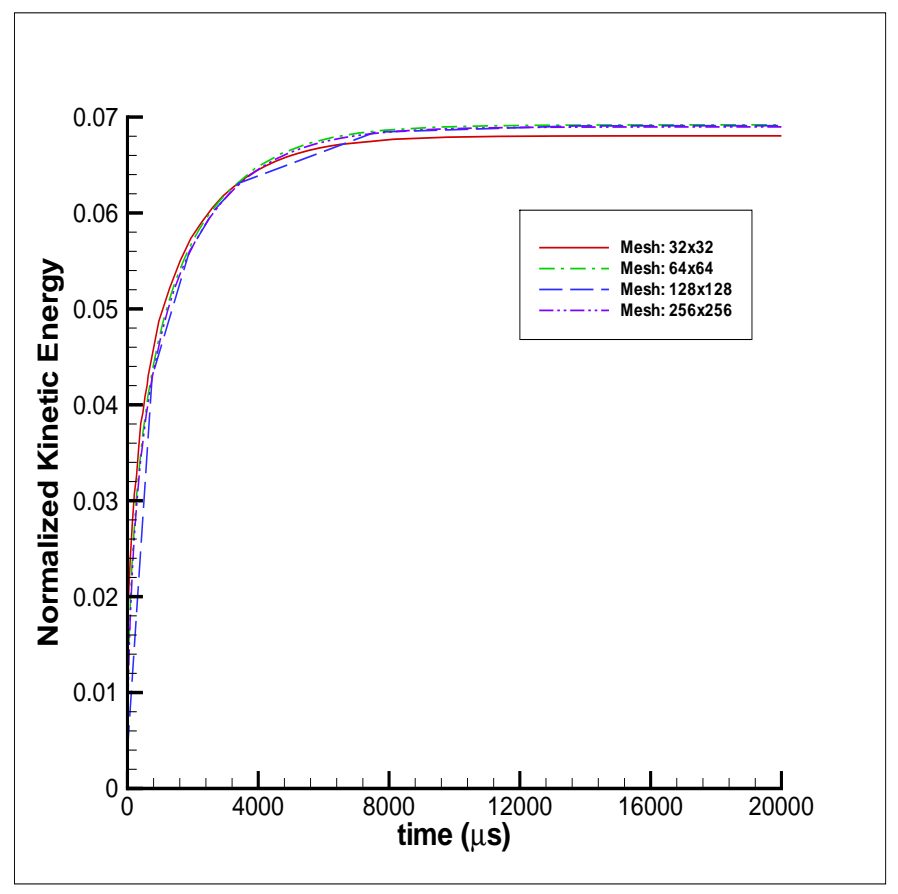

Figure 1: Time variation of the normalized kinetic energy for various mesh resolutions. The 2-D liddriven cavity problem is considered at $\mathrm{Re}=100$.

To showcase the results in a comprehensive manner, Tables 2 and 3 summarize the $(x, y)$-location of the primary vortex and the extrema of the velocity (along with their corresponding locations), respectively. Results from various published studies are also included. Ghia et al. [4] developed a second-order finite-difference method based on the vorticity- streamfunction formulation; while Deng et al. [3] ran computations using a finite-volume approach. Botella \& Peyret [2] performed simulations a spectal method with a polynomial of degree up to $N=96$. Hence, Botella \& Peyret [2] provided results with the most accurate approach for this verification case. The location of the primary vortex compares well with the data obtained by Ghia et al. [4]. For the extrema results, it is seen that at the highest resolution considered of $256 \times 256$, the current error in the minimum u-velocity is computed to be 0.03 percent; while the error in the minimum (maximum) v-velocity is found to be $7.3 \times 10^{-3}(0.055)$ percent. These 


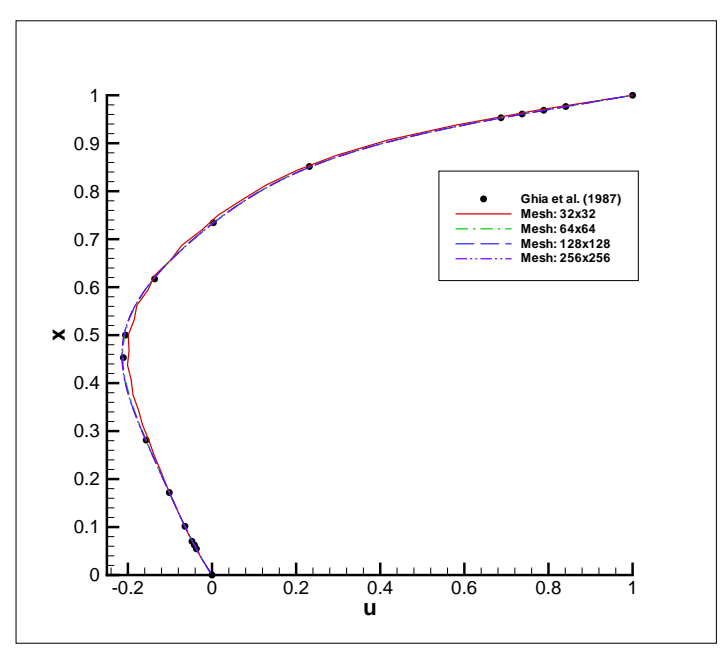

(a)

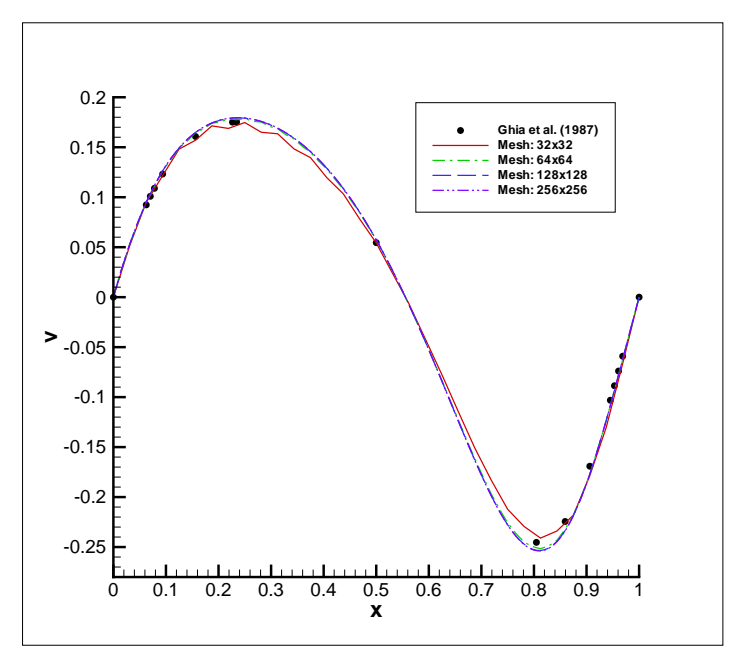

(b)

Figure 2: Distribution of (a) $u$ and (b) $v$-velocity along the centerlines for various mesh resolutions. The 2 -D lid-driven cavity problem is considered at $\mathrm{Re}=100$. Comparison with the results obtained by Ghia et al. $[4]$.

errors are computed by considering the current $A L E 3 D$ results and those obtained with the spectral method [2] using a polynomial of degree $N=96$. It should be noted that to compute these errors, the results on the various meshes have to achieve a criterion for convergence. Hence, the integrated quantity provides guidance on the quasi-steady state and its change between two instances should not be larger than $10^{-6}$. Once the simulations satisfy these criteria, the converged solution is compared to those obtained by published results.

\begin{tabular}{ccc}
\hline Reference & Grid & $(x, y)$ \\
\hline Ghia [4] & $129 \times 129$ & $(0.6172,0.7344)$ \\
Current & $32 \times 32$ & $(0.6250,0.7500)$ \\
& $64 \times 64$ & $(0.6250,0.734375)$ \\
& $128 \times 128$ & $(0.617188,0.742188)$ \\
& $256 \times 256$ & $(0.617188,0.738281)$ \\
\hline
\end{tabular}

Table 2: Location of the primary vortex for the 2 -D lid-driven cavity at $\operatorname{Re}=100$ 


\begin{tabular}{|c|c|c|c|c|}
\hline Reference & Grid & $\begin{array}{c}u_{\min } \\
{\left[x_{\min }\right]}\end{array}$ & $\begin{array}{c}v_{\max } \\
{\left[y_{\max }\right]}\end{array}$ & $\begin{array}{c}v_{\min } \\
{\left[y_{\min }\right]}\end{array}$ \\
\hline \multirow[t]{2}{*}{ Ghia [4] } & $129 \times 129$ & -0.21090 & 0.17527 & -0.24533 \\
\hline & & {$[0.4531]$} & {$[0.2344]$} & {$[0.8047]$} \\
\hline \multirow[t]{2}{*}{ Deng [3] } & $64 \times 64$ & -0.21315 & 0.17896 & -0.25339 \\
\hline & Extrapolation & -0.21405 & 0.17949 & -0.25399 \\
\hline \multirow[t]{2}{*}{ Botella \& Peyret [2] } & $N=96$ & -0.2140424 & 0.1795728 & -0.2538030 \\
\hline & & {$[0.4581]$} & {$[0.2370]$} & {$[0.8104]$} \\
\hline \multirow[t]{8}{*}{ Current } & $32 \times 32$ & -0.1962162 & 0.1676313 & -0.2261054 \\
\hline & & {$[0.46875]$} & {$[0.22875]$} & {$[0.78125]$} \\
\hline & $64 \times 64$ & -0.2102926 & 0.1786831 & -0.2516775 \\
\hline & & {$[0.453125]$} & {$[0.25000]$} & {$[0.8125]$} \\
\hline & $128 \times 128$ & -0.2139176 & 0.1794542 & -0.2534879 \\
\hline & & {$[0.460938]$} & {$[0.234375]$} & {$[0.8125]$} \\
\hline & $256 \times 256$ & -0.2141091 & 0.1795597 & -0.2538387 \\
\hline & & {$[0.457031]$} & {$[0.238281]$} & {$[0.808594]$} \\
\hline
\end{tabular}

Table 3: Extrema of the velocity and their corresponding locations through the centerlines of the cavity at $\operatorname{Re}=100$ 


\subsection{Air with $\gamma$-Law: $\operatorname{Re}=1000$}

This section describes results obtained from computations at $R e=1000$. A mesh resolution study is performed with grids consisting of $64 \times 64,128 \times 128,256 \times 256$ and $512 \times 512$ cells (or zones). Fig. 3 presents contours of the temperature field (computed from the internal energy) for Re $=1000$. Several key flow features are captured: a primary circulation zone in the center of the cavity; while two secondary vortices in the lower left and right corners. Further, a tertiary vortex is captured near the lower right corner. The center locations of these vortices have been extracted and compared with published results. Tables 4 and 5 summarize the $(x, y)$-location of the primary vortex and the extrema of the velocity (along with their corresponding locations), respectively. Results obtained by Ghia et al. [4] and Botella \& Peyret [2] are also provided. It is seen that at the highest resolution considered of $256 \times 256$, the current error in the minimum u-velocity is computed 0.47 percent; while the error in the minimum and maximum v-velocity is found to be 0.42 percent. These errors are computed by considering the current $A L E 3 D$ results and those obtained with the spectral method [2] using a polynomial of degree $N=160$.

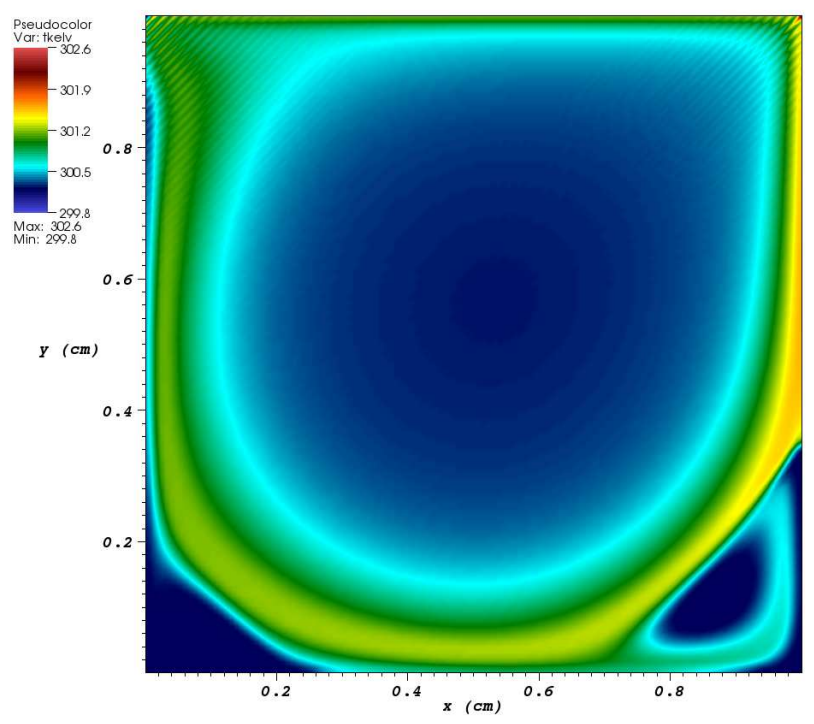

Figure 3: Contours of the temperature (based on the internal energy) for the 2-D lid-driven cavity problem at $\operatorname{Re}=1000$.

The locations of the lower left and right secondary vortices are presented in Table 6 and are compared with those obtained by [4] and [2]. It is seen that ALE3D captures quite well the small-scale features of the flow. Figs. 4(a) \& (b) plot the distributions of the $u$ and $v$-velocity along the vertical $(x=1 / 2)$ and horizontal $(y=1 / 2)$ centerlines for the various meshes considered. Also shown are the results obtained by Ghia et al. [4] and Botella \& Peyret [2]. The results obtained with ALE3D agree quite well with the published data. More quantitative data along select cell locations is presented below for $\operatorname{Re}=1000$. Tables 7 and 8 summarize the spatial distributions of the $u$ and $v$-velocities at select locations defined in Ghia et al. [4] along the vertical and horizontal centerlines, respectively. The values obtained by Ghia et al. [4] and Botella \& Peyret [2] are also shown. These tabulated values will be useful for future VERTS studies as new capabilities in $A L E 3 D$ are added and new developers/users familiarize themselves with 


\begin{tabular}{ccc}
\hline Reference & Grid & $(x, y)$ \\
\hline Ghia [4] & $129 \times 129$ & $(0.5313,0.5625)$ \\
Botella \& Peyret [2] & $N=160$ & $(0.5308,0.5652)$ \\
Current & $64 \times 64$ & $(0.5313,0.5625)$ \\
& $128 \times 128$ & $(0.5313,0.5625)$ \\
& $256 \times 256$ & $(0.5313,0.5664)$ \\
\hline
\end{tabular}

Table 4: Location of the primary vortex for the 2 -D lid-driven cavity at $\operatorname{Re}=1000$

\begin{tabular}{|c|c|c|c|c|}
\hline Reference & Grid & $\begin{array}{c}u_{\min } \\
{\left[x_{\min }\right]}\end{array}$ & $\begin{array}{c}v_{\max } \\
{\left[y_{\max }\right]}\end{array}$ & $\begin{array}{l}v_{\min } \\
{\left[y_{\min }\right]}\end{array}$ \\
\hline Ghia [4] & $129 \times 129$ & $\begin{array}{c}-0.38289 \\
{[0.1719]}\end{array}$ & $\begin{array}{l}0.37095 \\
{[0.1563]}\end{array}$ & $\begin{array}{c}-0.51550 \\
{[0.9063]}\end{array}$ \\
\hline Deng [3] & $\begin{array}{c}\qquad 128 \times 128 \\
\text { Extrapolation }\end{array}$ & $\begin{array}{l}-0.38511 \\
-0.38867\end{array}$ & $\begin{array}{l}0.37369 \\
0.37702\end{array}$ & $\begin{array}{l}-0.52280 \\
-0.52724\end{array}$ \\
\hline Botella \& Peyret [2] & $N=160$ & $\begin{array}{c}-0.3885698 \\
{[0.1717]}\end{array}$ & $\begin{array}{c}0.3769447 \\
{[0.1578]}\end{array}$ & $\begin{array}{c}-0.5270771 \\
{[0.9092]}\end{array}$ \\
\hline Current & $64 \times 64$ & $\begin{array}{c}-0.3484267 \\
{[0.1875]}\end{array}$ & $\begin{array}{c}0.4036732 \\
{[0.1875]}\end{array}$ & $\begin{array}{c}-0.4829184 \\
{[0.9063]}\end{array}$ \\
\hline & $128 \times 128$ & $\begin{array}{c}-0.3792116 \\
{[0.1719]}\end{array}$ & $\begin{array}{r}0.3688710 \\
{[0.15625]}\end{array}$ & $\begin{array}{c}-0.5163308 \\
{[0.9063]}\end{array}$ \\
\hline & $256 \times 256$ & $\begin{array}{c}-0.38675684 \\
{[0.1719]}\end{array}$ & $\begin{array}{l}0.375343 \\
{[0.16016]}\end{array}$ & $\begin{array}{c}-0.524852 \\
{[0.9102]}\end{array}$ \\
\hline
\end{tabular}

Table 5: Extrema of the velocity and their corresponding locations through the centerlines of the cavity at $R e=1000$ 
the code.

\begin{tabular}{cccc}
\hline Reference & Grid & $\begin{array}{c}(x, y)_{\text {Secondary }} \\
(\text { Lower Left })\end{array}$ & $\begin{array}{c}(x, y)_{\text {Secondary }} \\
\text { (Lower Right) }\end{array}$ \\
\hline Ghia [4] & $129 \times 129$ & $(0.0859,0.0781)$ & $(0.8594,0.1094)$ \\
Botella \& Peyret [2] & $N=160$ & $(0.0833,0.0781)$ & $(0.8640,0.1118)$ \\
Current & $64 \times 64$ & $(0.0938,0.0625)$ & $(0.8906,0.1250)$ \\
& $128 \times 128$ & $(0.0820,0.0781)$ & $(0.8633,0.1094)$ \\
& $256 \times 256$ & $(0.0820,0.0781)$ & $(0.8652,0.1133)$ \\
& & &
\end{tabular}

Table 6: Locations of the secondary vortex at lower left and lower right corners for the 2-D lid-driven cavity at $\operatorname{Re}=1000$.

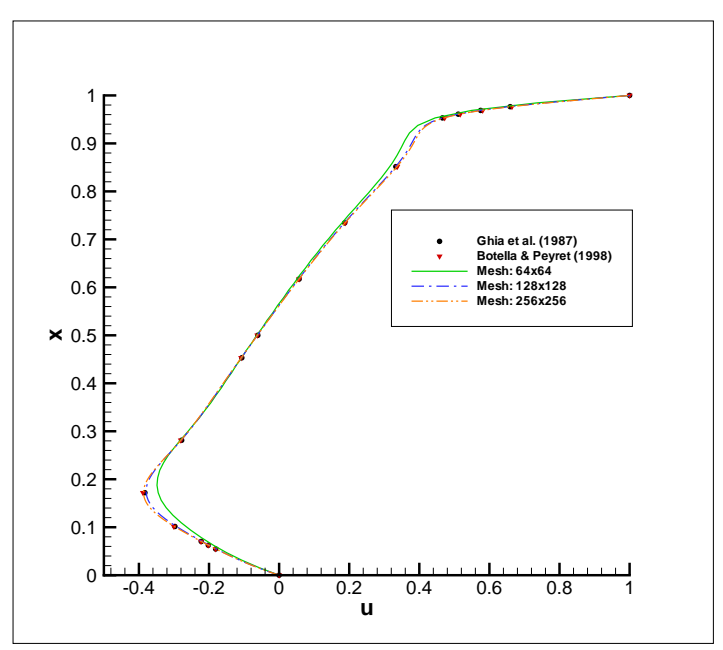

(a)

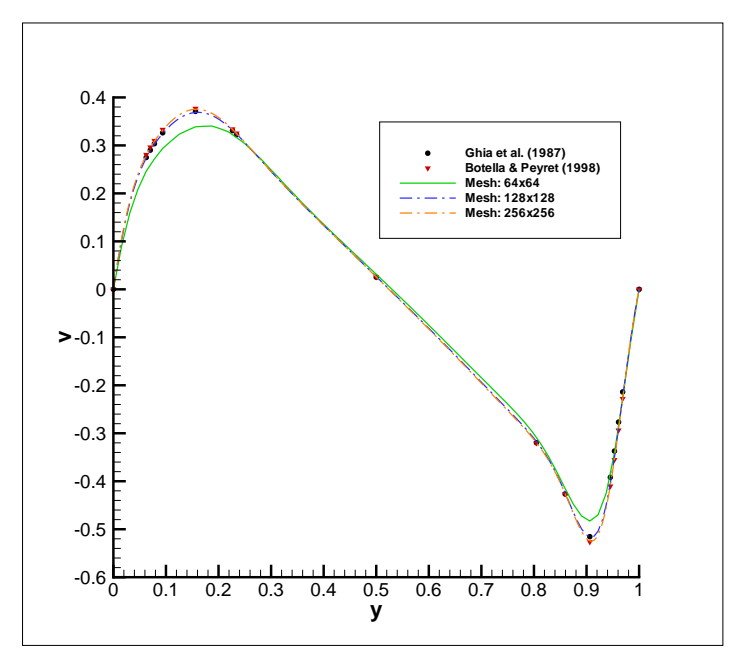

(b)

Figure 4: Distribution of (a) $u$ and (b) $v$-velocity along the centerlines for various mesh resolutions. The 2-D lid-driven cavity problem is considered at $\mathrm{Re}=1000$. Comparison with the results obtained by Ghia et al. [4] and Botella \& Peyret[2]. 


\begin{tabular}{|c|c|c|c|c|}
\hline$y$ & $u$ & $u$ & $u$ & $u$ \\
\hline & Ghia [4] & Botella [2] & $\begin{array}{l}128 \times 128 \\
\text { (Current) }\end{array}$ & $\begin{array}{l}256 \times 256 \\
(\text { Current) }\end{array}$ \\
\hline 0.0000 & 0.00000 & 0.0000000 & 0.0000000 & 0.0000000 \\
\hline 0.0547 & -0.18109 & -0.1812881 & -0.1791172 & -0.1805828 \\
\hline 0.0625 & -0.20196 & -0.2023300 & -0.1995642 & -0.2015452 \\
\hline 0.0703 & -0.22220 & -0.2228955 & -0.2194107 & -0.2220199 \\
\hline 0.1016 & -0.29730 & -0.3004561 & -0.2930721 & -0.2988949 \\
\hline 0.1719 & -0.38289 & -0.3885691 & -0.3792116 & -0.3867568 \\
\hline 0.2813 & -0.27805 & -0.2803696 & -0.2797030 & -0.8025292 \\
\hline 0.4531 & -0.10648 & -0.1081999 & -0.1078106 & -0.1081180 \\
\hline 0.5000 & -0.06080 & -0.0620561 & -0.0621722 & -0.0620909 \\
\hline 0.6172 & 0.05702 & 0.0570178 & 0.0558182 & 0.0567525 \\
\hline 0.7344 & 0.18719 & 0.1886747 & 0.1863240 & 0.1881635 \\
\hline 0.8516 & 0.33304 & 0.3372212 & 0.3325964 & 0.3362661 \\
\hline 0.9531 & 0.46604 & 0.4723329 & 0.4600416 & 0.4711001 \\
\hline 0.9609 & 0.51117 & 0.5169277 & 0.5097545 & 0.5155230 \\
\hline 0.9688 & 0.57492 & 0.5808359 & 0.5719110 & 0.5784138 \\
\hline 0.9766 & 0.65928 & 0.6644227 & 0.6549291 & 0.6618858 \\
\hline 1.0000 & 1.00000 & 1.0000000 & 1.0000000 & 1.0000000 \\
\hline
\end{tabular}

Table 7: Distribution of the $\mathrm{u}$-velocity through the vertical centerline of the cavity $(x=1 / 2)$ at $\operatorname{Re}=1000$ provided at locations given in [4]. The mesh resolutions are $129 \times 129$ nodes, $N=160$ polynomial degree and $128^{2} \& 256^{2}$ cells for Ghia et al. [4], Botella \& Peyret [2] and current study, respectively. 


\begin{tabular}{|c|c|c|c|c|}
\hline$x$ & $v$ & $v$ & $v$ & $v$ \\
\hline & Ghia [4] & Botella [2] & $128 \times 128$ & $256 \times 256$ \\
\hline & & & (Current) & (Current) \\
\hline 0.0000 & 0.00000 & 0.0000000 & 0.0000000 & 0.0000000 \\
\hline 0.0625 & 0.27485 & 0.2807056 & 0.2739369 & 0.2795766 \\
\hline 0.0703 & 0.29012 & 0.2962703 & 0.2889852 & 0.2950828 \\
\hline 0.0781 & 0.30353 & 0.3099097 & 0.3025024 & 0.3086624 \\
\hline 0.0938 & 0.32627 & 0.3330442 & 0.3249063 & 0.3315471 \\
\hline 0.1563 & 0.37095 & 0.3769189 & 0.3688710 & 0.3753322 \\
\hline 0.2266 & 0.33075 & 0.3339924 & 0.3318112 & 0.3334892 \\
\hline 0.2344 & 0.32235 & 0.3253592 & 0.3237234 & 0.3249170 \\
\hline 0.5000 & 0.02526 & 0.0257995 & 0.0266271 & 0.0258745 \\
\hline 0.8047 & -0.31966 & -0.3202137 & -0.3171915 & -0.3196104 \\
\hline 0.8594 & -0.42665 & -0.4264545 & -0.4244512 & -0.4259453 \\
\hline 0.9063 & -0.51550 & -0.5264392 & -0.5163308 & -0.5243499 \\
\hline 0.9453 & -0.39188 & -0.4103754 & -0.4047734 & -0.4089855 \\
\hline 0.9531 & -0.33714 & -0.3553213 & -0.3508494 & -0.3539418 \\
\hline 0.9609 & -0.27669 & -0.2936869 & -0.2900014 & -0.2922751 \\
\hline 0.9688 & -0.21388 & -0.2279225 & -0.2257287 & -0.2273293 \\
\hline 1.0000 & 0.00000 & 0.0000000 & 0.0000000 & 0.0000000 \\
\hline
\end{tabular}

Table 8: Distribution of the v-velocity through the horizontal centerline of the cavity $(y=1 / 2)$ at $\operatorname{Re}=1000$ provided at locations given in [4]. The mesh resolutions are $129 \times 129$ nodes, $N=160$ polynomial degree and $128^{2} \& 256^{2}$ cells for Ghia et al. [4], Botella \& Peyret [2] and current study, respectively. 


\subsection{Melted Metals with LEOS: $\mathrm{Re}=1000$}

Here, the 2-D lid-driven cavity will be considered with working liquid as melted metals, including aluminum and copper, instead of air. Although no proper comparison is currently available in the literature for these types of liquids, we will perform a similar analysis to that conducted for air. The objective is to gain further confidence in the results obtained with $A L E 3 D$ when using different strength and material models. Computations for the 2 -D lid-driven cavity at $\mathrm{Re}=1000$ will be pursued. A key issue encountered at problem sett-up is to maintain similarity of two non-dimensional variables. These parameters are Reynolds number (representing the ratio of advection to viscous effects) and Mach number (corresponding to compressiblity effects); mathematically this means:

$$
\begin{array}{r}
R e_{\text {Air }}=R e_{\text {Melted Metal }} \\
M a c h_{\text {Air }}=M a c h_{\text {Melted Metal }}
\end{array}
$$

A constant Mach number with a value of 0.0448091 is selected with $\mathrm{Re}=1000$. Hence, the length of the cavity is modified (typically decreases) compared to the case with air; further, the top wall velocity is changed accordingly. Table 9 shows the values for the cavity height and the top-wall velocity considered for the cases with melted metals. These values corresponds to a configuration with air at similar Re and Ma. The 2-D lid-driven cavity for air has a length of $h_{\text {Air }}=0.1 \mathrm{~cm}$ and a top-wall velocity $\mathrm{U}_{\text {top,Air }}=0.00148333 \mathrm{~cm} / \mu \mathrm{s}$. The values in the LEOS table used are 130 and 290 for $\mathrm{Al}$ and $\mathrm{Cu}$, respectively.

\begin{tabular}{ccc}
\hline Metal & $\mathrm{h}$ & $\mathrm{U}_{\text {top }}$ \\
& $(\mu \mathrm{m})$ & $(\mathrm{cm} / \mu \mathrm{s})$ \\
\hline Aluminum & 3.77538 & 0.0211454 \\
Copper & 2.95077 & 0.0154043
\end{tabular}

Table 9: Simulation matrix considered for the 2-D lid-driven cavity using melted metals.

Table 10 summarizes the various test cases for the melted metals. Iinitially, the computations are compared with melted Aluminum $(\mathrm{Al})$ and Copper $(\mathrm{Cu})$ using a simple EOS. Figs. 5(a) \& (b) show the distribution of the $u$ and $v$-velocity along the vertical $(x=1 / 2)$ and horizontal $(y=1 / 2)$ centerlines for a mesh of $128 \times 128$ cells. Also plotted are the results obtained by Ghia et al. [4] and Botella \& Peyret[2].It is seen that the results are similar for both metals. Hence, we will only consider in this study the simulations obtained with melted Aluminum. For the results discussed below, the EOS is based on an LEOS table look-up available in $A L E 3 D$.

Tables 11 and 12 summarize the $(x, y)$-location of the primary vortex and the extrema of the velocity (along with their corresponding locations), respectively. Results obtained by Ghia et al. [4] and Botella \& Peyret [2] are also provided. The locations of the lower left and right secondary vortices are shown in Table 13. The results are compared with those obtained by [4] and [2]. It seen that $A L E 3 D$ captures quite well the small-scale features of the flow. Hence, these tabulated values are provided for future VERTS analysis. Figs. 6(a) \& (b) show the distributions of the $u$ and $v$-velocity along the vertical $(x=1 / 2)$ and horizontal $(y=1 / 2)$ centerlines for the various meshes considered. Also plotted are the results obtained by Ghia et al. [4] and Botella \& Peyret [2]. The agreement with the published results is qualitatively appropriate with the overall trend matching the published data. As anticipated, the peak 


\begin{tabular}{ccc}
\hline Metal & Re & Mesh \\
& & Resolution \\
& & \\
\hline Aluminum & 1000 & $64 \times 64,128 \times 128$ \\
& & $256 \times 256,512 \times 512$ \\
Copper & 1000 & $128 \times 128$ \\
\hline
\end{tabular}

Table 10: Simulation matrix considered for the 2-D lid-driven cavity using melted metals.

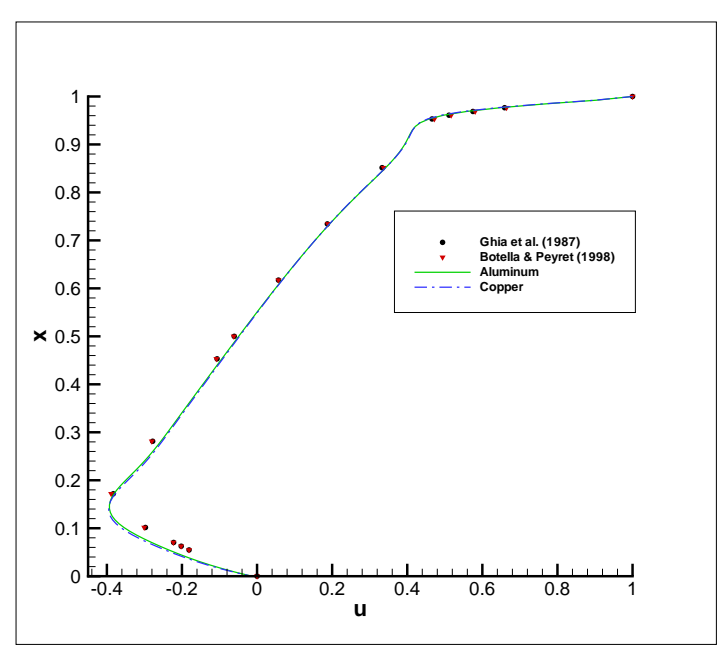

(a)

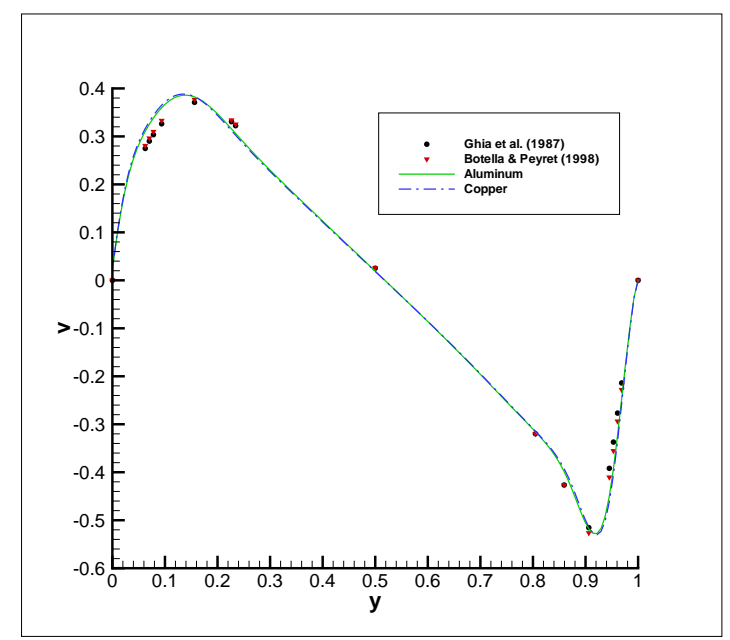

(b)

Figure 5: Distribution of (a) $u$ and (b) $v$-velocity along the centerlines for melted $\mathrm{Al}$ and $\mathrm{Cu}$ at $\mathrm{Re}=1000$. Comparison with the results obtained by Ghia et al.[4] and Botella \& Peyret[2].

velocity (and their respective locations) have a shift when compared with the simulations obtained for an incompressible fluid. 


\begin{tabular}{ccc}
\hline Reference & Grid & $(x, y)$ \\
\hline Ghia [4] & $129 \times 129$ & $(0.5313,0.5625)$ \\
Botella \& Peyret [2] & $N=160$ & $(0.5308,0.5652)$ \\
Current & $64 \times 64$ & $(0.5312,0.5625)$ \\
& $128 \times 128$ & $(0.5234,0.5547)$ \\
& $256 \times 256$ & $(0.5273,0.5547)$ \\
\hline
\end{tabular}

Table 11: Location of the primary vortex for the 2-D lid-driven cavity for melted $\mathrm{Al}$ at $\mathrm{Re}=1000$

\begin{tabular}{|c|c|c|c|c|}
\hline Reference & Grid & $\begin{array}{c}u_{\min } \\
{\left[x_{\min }\right]}\end{array}$ & $\begin{array}{c}v_{\max } \\
{\left[y_{\max }\right]}\end{array}$ & $\begin{array}{c}v_{\min } \\
{\left[y_{\min }\right]}\end{array}$ \\
\hline \multirow[t]{2}{*}{ Ghia [4] } & $129 \times 129$ & -0.38289 & 0.37095 & -0.51550 \\
\hline & & [0.1719] & [0.1563] & [0.9063] \\
\hline \multirow[t]{2}{*}{ Deng [3] } & $128 \times 128$ & -0.38511 & 0.37369 & -0.52280 \\
\hline & Extrapolation & -0.38867 & 0.37702 & -0.52724 \\
\hline \multirow[t]{2}{*}{ Botella \& Peyret [2] } & $N=160$ & -0.3885698 & 0.3769447 & -0.5270771 \\
\hline & & [0.1717] & [0.1578] & [0.9092] \\
\hline \multirow[t]{6}{*}{ Current } & $64 \times 64$ & -0.3535933 & 0.3487934 & -0.4860469 \\
\hline & & [0.1719] & [0.1563] & [0.9219] \\
\hline & $128 \times 128$ & -0.391704 & 0.3848114 & -0.5289330 \\
\hline & & [0.1484] & {$[0.1406]$} & [0.9219] \\
\hline & $256 \times 256$ & -0.4015147 & 0.3931526 & -0.5382188 \\
\hline & & [0.1406] & [0.1367] & [0.9219] \\
\hline
\end{tabular}

Table 12: Extrema of the velocity and their corresponding locations through the centerlines of the cavity for melted $\mathrm{Al}$ at $\mathrm{Re}=1000$. 


\begin{tabular}{cccc}
\hline Reference & Grid & $\begin{array}{c}(x, y)_{\text {Secondary }} \\
(\text { Lower Left })\end{array}$ & $($ Lower Right $)$ \\
& & & \\
\hline Ghia [4] & $129 \times 129$ & $(0.0859,0.0781)$ & $(0.8594,0.1094)$ \\
Botella \& Peyret [2] & $N=160$ & $(0.0833,0.0781)$ & $(0.8640,0.1118)$ \\
Current & $64 \times 64$ & $(0.0938,0.0781)$ & $(0.8906,0.1094)$ \\
& $128 \times 128$ & $(0.0859,0.0859)$ & $(0.8516,0.1017)$ \\
& $256 \times 256$ & $(0.0859,0.0898)$ & $(0.8555,0.1055)$
\end{tabular}

Table 13: Location of the lower left secondary vortex for the 2-D lid-driven cavity for melted $\mathrm{Al}$ at $\operatorname{Re}=1000$.

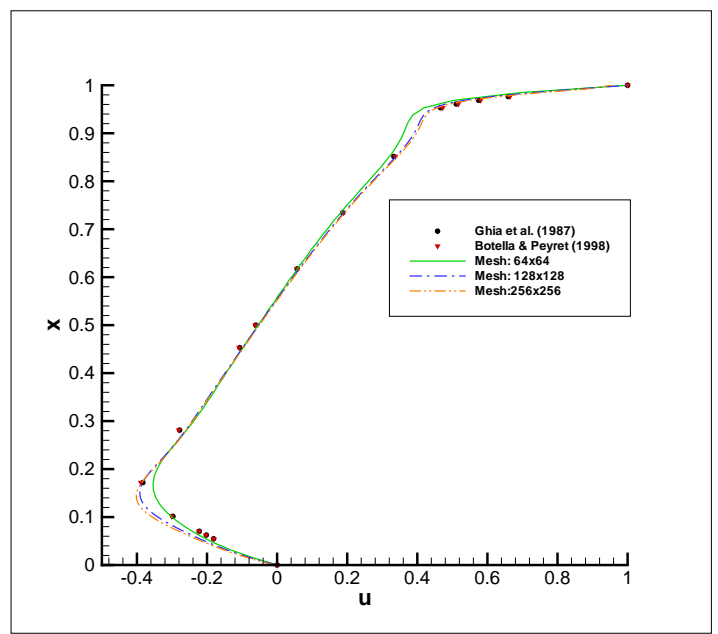

(a)

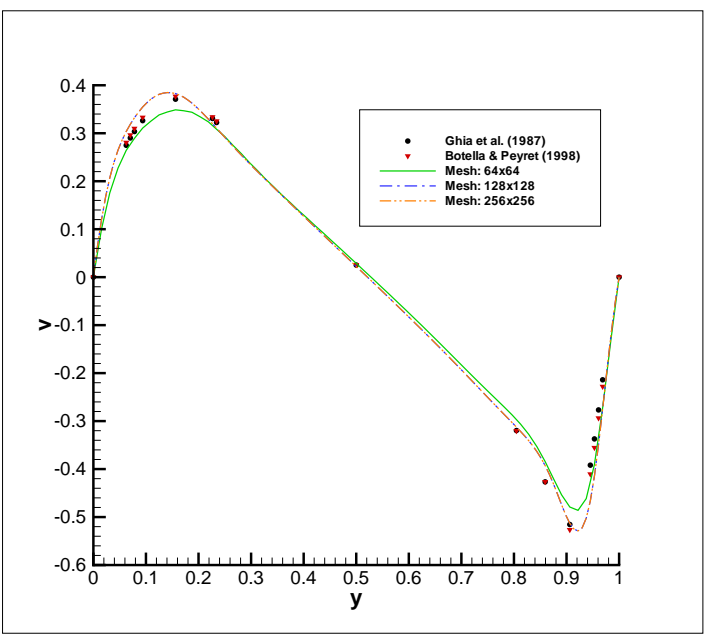

(b)

Figure 6: Distribution of (a) $u$ and (b) $v$-velocity along the centerlines for melted $\mathrm{Al}$ at $\mathrm{Re}=1000$ for various mesh resolutions. Comparison with the results obtained by Ghia et al. [4] and Botella \& Peyret [2].

\section{Verification Test Case for Hydro-Thermal Applications: 2-D Steady Laminar Couette Flow}

The second test case performed is the 2-D steady laminar Couette flow. The geometry consists of a rectangular configuration with a height of $25 \mu \mathrm{m}$ and a length of $500 \mu \mathrm{m}$. The specified boundary conditions are a no-slip velocity on the bottom wall, a specified slip velocity on the top wall and a 
linearly varying inflow velocity at the left boundary with a non-reflective outflow boundary on the right. The mesh resolution is $500 \times 25$ zones of uniform size. This results in a cell size of $\Delta x=\Delta y=1 \mu \mathrm{m}$. The top velocity is set to $U_{\text {Top }}=0.00319328 \mathrm{~cm} / \mu \mathrm{s}$, resulting in a Reynolds number of 1000 . Since the flow transitions to turbulence at $\mathrm{Re}=2000$, the laminar regime presides for this test case. A simple analytical solution for the velocity field can be easily derived and is $u / U_{t o p}=y / h$. This means that the $z$-component of vorticity, defined as $\omega_{z}=\frac{\partial v}{\partial x}-\frac{\partial u}{\partial y}=-U / h$, is constant. The area-weighted spanwise vorticity field is chosen to assess when the flow reaches a quasi-steady state. The criterion is based on a difference of less than $10^{-6}$ between two time instances. Once this is achieved, the results obtained from the simulation are compared to the analytical field. An error for the $u$-velocity of $4.93 \times 10^{-9}$ was obtained. It was seen that the y-distribution of the $\omega_{z}$ variation is quite constant along the channel height (as anticipated) with a mean value of $-1.2773117 \mu^{-1}$ comparing quite well with the analytical value of $-1.2773108 \mu \mathrm{s}^{-1}$. The $\mathrm{L}_{2}$ error-norm is computed to be $2.8575287 \times 10^{-6}$.

Further, the thermal solver available in $A L E 3 D$ is activated to verify the accuracy of the temperature field. For the current Couette problem, the steady governing equations for the thermal field consists of the balance between the conductivity term and viscous dissipation as follows:

$$
k \frac{d^{2} T}{d y^{2}}+\mu\left(\frac{d u}{d y}\right)^{2}=0
$$

Hence, the temperature field has the following analytical form:

$$
T=\frac{\phi}{2 k}(h-y) y+T_{W a l l}
$$

where $\phi=\mu U_{\text {top }}^{2} / h^{2}$ corresponds to the viscous dissipation, $T_{W \text { all }}$ is the prescribed temperature on the top and bottom walls. Similar configuration to the hydro component described above is used where the top and bottom temperatures are set to 950K. Fig. 7 presents the $y$-variation of the temperature along the height for various mesh resolutions in the $y$-direction, ranging from 20 to 80 zones. Also shown is the distribution of the analytical field. It is observed the parabolic profile is well captured. Table 14 summarizes the values of the $\mathrm{L}_{2}$ error-norms at increasing resolution. We determined the formal order of accuracy $\lambda=\log \left(\frac{E_{1}}{E 2}\right) / \log \left(\frac{\Delta y_{1}}{\Delta y_{2}}\right)$. Hence, an overall accuracy of $\lambda \sim 1.9$ is achieved for the thermal solver.

\begin{tabular}{ccc}
\hline$N_{y}$ & Error & $\lambda$ \\
\hline 20 & $6.5883023 \times 10^{-4}$ & \\
40 & $1.822891 \times 10^{-4}$ & 1.85 \\
80 & $4.698138 \times 10^{-5}$ & 1.96
\end{tabular}

Table 14: Computed $\mathrm{L}_{2}$ error norm for the temperature field in a thermal Couette flow.

\section{Conclusions}

A verification test suite has been assessed with primary focus on rail gun applications. The computational multiphysics framework, ALE3D, is used. The focus of the current study is to provide guidance and 


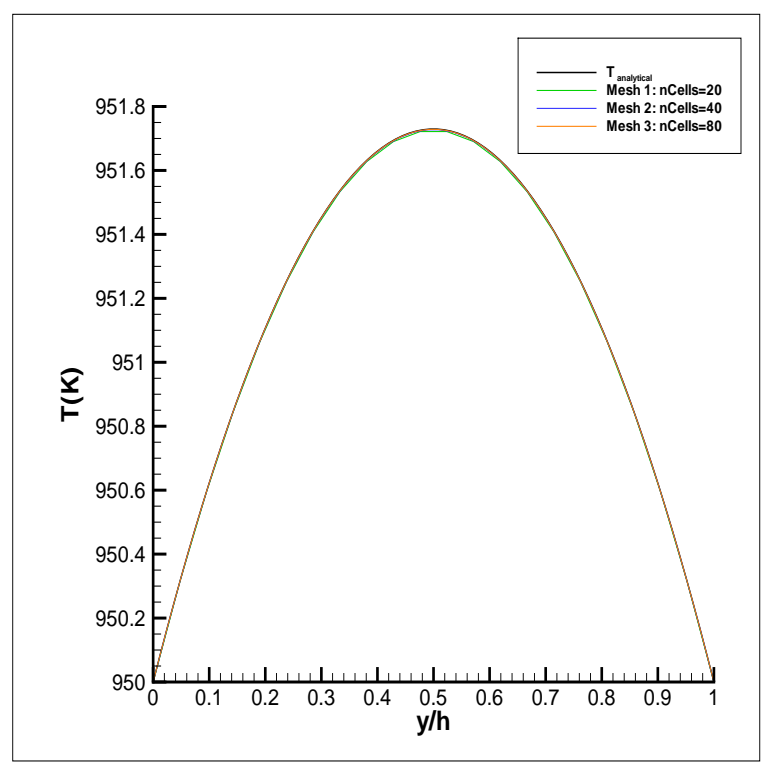

Figure 7: Distribution of temperature along the channel height for a Couette flow at $\operatorname{Re}=1000$.

confidence for the results obtained with $A L E 3 D$. Several 2-D test cases including the lid-driven cavity and the Couette flow are investigated. The results are compared with analytical solutions and previously published results. Mesh resolution studies are performed along with various models for the equation of state. The next phase will include Electromagnetic (EM) component and a VERTS analysis will be undertaken with the Hydro-Thermal-EM modules being coupled.

\section{Acknowledgments}

This research was supported by Navy Railgun Bore Life Consortium. The authors acknowledge numereous discussions with Drs. R. McCallen, and K Salari. Mr. T. Dunn kindly provided the Python script to extract lineout data and his contribution is appreciated.

\section{References}

[1] User's Manual for ALE3D: An Arbitrary Lagrangian-Eulerian 3D Code System Vols 1 \& 2. LLNL. Version 4.8 (2008)

[2] Botella, O., \& Peyret, R. Benchmark Spectral Results on the Lid-Driven Cavity Flow.Computers E Fluids, Vol. 27, No. 4, $421-433$ (1998)

[3] Deng, G.B., Piquet, J., Queutey, P., \& Visonneau, M. Incompressible Flow Calculations with a Consistent Physical Interpolation Finite Volume Approach.Computers 83 Fluids, Vol. 23, No. 8, 1029- 1047 (1994)

[4] Ghia, U., Ghia, N. \& Shin, C.T. High-Re Solutions for Incompressible Flow using the Navier-Stokes Equations and a Multigrid Method.Journal of Computational Physics, Vol. 48, 387 - 411 (1987)

[5] Oberkampf, W.L., \& Trucano T.G. Verification and Validation in Computational Fluid Dynamics. Progress in Aerospace Sciences Vol. 38, 209 - 272 ( (2002) 
[6] Oberkampf, W.L., Trucano T.G., \& Hirsch, C. Verification, Validation and Predictive Capability in Computational Engineering and Physics. Applied Mechanics Reviews Vol. 57, No. 5, 345 - 384 ( (2003)

[7] Trucano T.G. \& Garasi, C.J. Verification and Validation for Railgun Bore Life Computational Modeling and Simulation. Sandia Report, SAND2007-4256 (2007) 